\title{
Short- and long-term outcomes of endoscopic submucosal dissection for non-ampullary duodenal neuroendocrine tumors
}

\author{
Masafumi Nishio a, Kingo Hirasawa ${ }^{a}$, Yuichiro Ozekia ${ }^{a}$ Atsushi Sawada ${ }^{a}$, Ryosuke Ikeda ${ }^{a}$, \\ Takehide Fukuchi ${ }^{a}$, Ryosuke Kobayashia, Makomo Makazua , Chiko Sato ${ }^{a}$, Shin Maedab \\ Yokohama City University Medical Center; Yokohama City University Graduate School of Medicine, Japan
}

Abstract

\section{Introduction}

The gastrointestinal tract is the most frequent site of neuroendocrine tumors (NETs) [1]. Duodenal NETs occur less frequently than gastric and rectal NETs, accounting for $<5 \%$

${ }^{a}$ Division of Endoscopy, Yokohama City University Medical Center (Masafumi Nishio, Kingo Hirasawa, Yuichiro Ozeki, Atsushi Sawada, Ryosuke Ikeda, Takehide Fukuchi, Ryosuke Kobayashi, Makomo Makazu, Chiko Sato); b Department of Gastoenterology, Yokohama City University Guraduate School of Medicine (Shin Maeda), Japan

Conflict of Interest: None

Correspondence to: Kingo Hirasawa, Division of Endoscopy, Yokohama City University Medical Center, 4-57 Urafune-cho, Minami-ku,

Yokohama 232-0024, Japan, e-mail: kingo-h@urahp.yokohama-cu.ac.jp

Received 17 December 2019; accepted 11 March 2020;

published online 13 April 2020

DOI: https://doi.org/10.20524/aog.2020.0477 of the total number $[1,2]$. However, these tumors are being recognized at an increasing frequency with the widespread use of gastrointestinal screening endoscopy [3].

According to the European Neuroendocrine Tumor Society guidelines, endoscopic resection is recommended for non-ampullary duodenal neuroendocrine tumors (NAD$\mathrm{NET}) \leq 10 \mathrm{~mm}$ in diameter and confined to the submucosal layer, without lymph node or distant metastasis [4,5]. Various methods of endoscopic resection, including endoscopic mucosal resection (EMR), EMR with ligation device (EMR-L), EMR with circumferential precutting (EMR-P), cap-assisted EMR (EMR-C), and endoscopic submucosal dissection (ESD), have been reported in previous studies [6-18]. However, there is no consensus regarding the preferred method of endoscopic resection for NAD-NET.

Previous studies have reported that ESD was superior to EMR for achieving en bloc, R0 and curative resections of rectal NETs [19-21]. ESD for NAD-NETs is reportedly technically difficult and associated with a high frequency of complications; 
however, it achieves better en bloc and curative resection rates than EMR [13-18]. Since there are only a few published studies with a very small number of patients, we aimed to assess the efficacy and safety of ESD for NAD-NET over a longer period and with a slightly higher number of cases.

\section{Patients and methods}

\section{Enrolled patients and tumors}

Between January 2015 and September 2018, 8 consecutive patients with 8 NAD-NETs underwent ESD at Yokohama City University Medical Center. In all cases, esophagogastroduodenoscopy (EGD), endoscopic ultrasound (EUS; high-frequency miniprobe, UM-2R, 20MHz; Olympus, Tokyo, Japan), and computed tomography (CT) were performed before ESD. We confirmed that all patients met the following criteria before ESD: i) histological diagnosis of NET G1 via endoscopic biopsy; ii) tumor $\leq 10 \mathrm{~mm}$ in diameter on EUS; iii) confined to submucosal layer on EUS; and iv) no regional lymph node enlargement or distant metastasis on CT. The procedures were performed in accordance with the Helsinki Declaration of the World Medical Association. exclusive pump (Telfusion pump; TERUMO, Tokyo, Japan) and pentazocine $(15 \mathrm{mg})$. A single-channel upper gastrointestinal endoscope with a water-jet system (GIF-Q260J; Olympus) was used. Several spots were marked at least $5 \mathrm{~mm}$ outside the border of the lesion with the Dual knife (Olympus). After injection of $0.4 \%$ hyaluronic acid solution (MucoUp; Johnson \& Johnson Medical Co., Tokyo, Japan) into the submucosa, the mucosal incision was performed outside of the markings using the Dual knife to achieve negative horizontal margins. After mucosal incision, submucosal dissection was also performed using the Dual knife $(1.5 \mathrm{~mm})$. To achieve negative vertical margins, submucosal dissection was performed as close to the muscle layer as possible. A high-frequency generator (VIO 300D; ERBE, Tübingen, Germany) was used during mucosal incision and submucosal dissection: mucosal incision was performed using ENDO CUT I mode (Effect 2), and submucosal dissection was performed using SWIFT COAG mode (Effect 3, 40W). Carbon dioxide insufflation was used during all ESD cases. In 7 cases, the artificial ulcer that developed after ESD was covered with a polyglycolic acid (PGA) sheet (Neoveil; Gunze Co., Kyoto, Japan) and fixed in place with fibrin glue (Beriplast P Combi-Set; CSL Behring Pharma, Tokyo, Japan) to prevent delayed perforation. All procedures were performed by an experienced endoscopist who had previously performed more than 20 duodenal ESDs for epithelial tumors (Fig. 1).

\section{ESD procedures}

All patients underwent ESD under sedation with intravenous propofol $(0.8-2.0 \mathrm{mg} / \mathrm{kg} / \mathrm{h})$ administered using an

\section{Histological evaluation}

After fixing in $10 \%$ formalin and serial sectioning at 2-mm intervals, the resected specimens were assessed
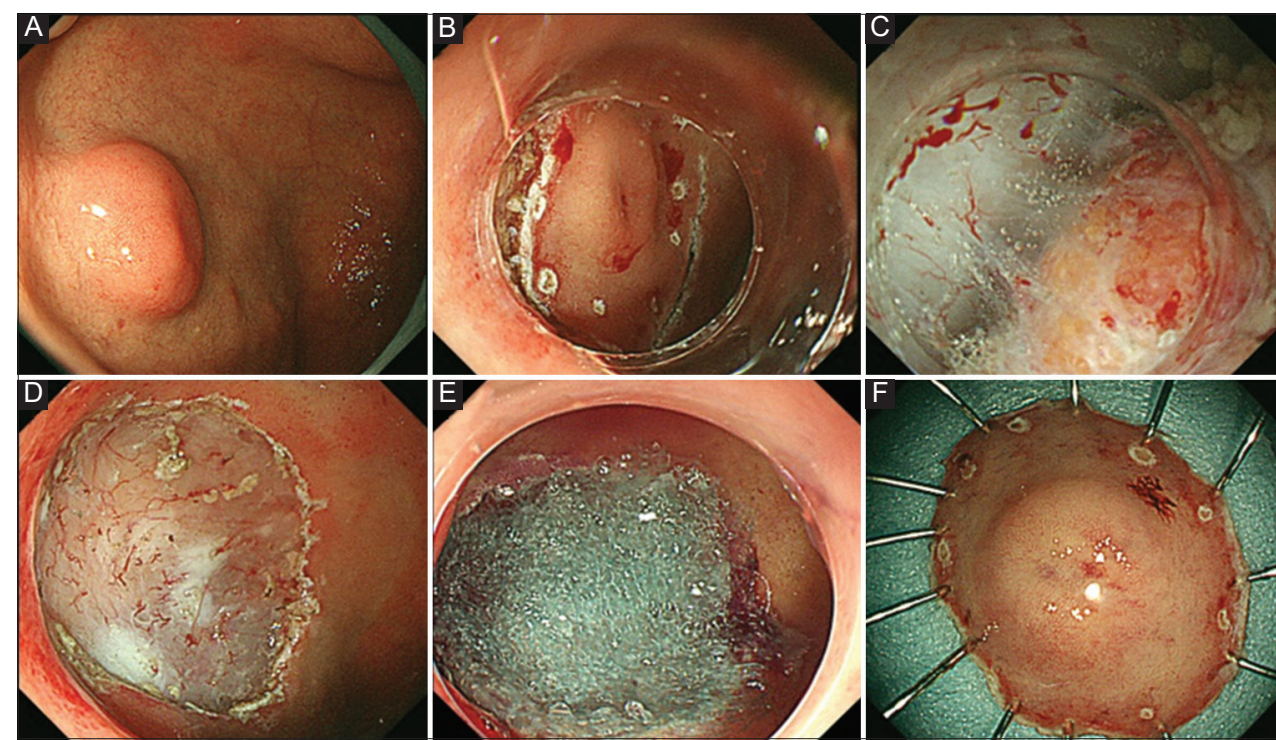

Figure 1 Endoscopic submucosal dissection technique. (A) A non-ampullary duodenal neuroendocrine tumor is observed in the anterior wall of the duodenal bulb. (B) Mucosal incision is performed using the Dual knife after marking with dots around the tumor. (C) Submucosal dissection is performed using the Dual knife $(1.5 \mathrm{~mm})$ as close to the muscle layer as possible. Since few Brunner's glands exist just below the tumor, we should be careful not to injure the tumor when submucosal dissection is at that site. In contrast, when the lifting of the submucosal injection around the tumor is insufficient because of abundant Brunner's glands, we should be careful not to injure the muscle layer to avoid intraoperative perforation. (D) The tumor is completely removed with no intraoperative perforation. (E) The artificial ulcer after endoscopic submucosal dissection is covered with a polyglycolic acid sheet, fixed in place with fibrin. (F) The tumor is resected en bloc 
histologically. Experienced gastrointestinal pathologists assessed the histological type, macroscopic appearance, tumor size, depth of invasion, lymphatic and vascular involvement, and horizontal and vertical margins. For classification of histological type, the World Health Organization (WHO) 2010 classification of tumors of digestive system was used [22]. Immunohistochemical analysis with 2 well-characterized neuroendocrine markers, chromogranin A and synaptophysin, was performed to reach an accurate diagnosis. In addition, the $\mathrm{Ki}-67$ index was assessed in all cases to classify tumors as G1, G2, or G3 according to the WHO classification.

\section{Definition}

En bloc resection was defined as resection of the lesion in a single piece with no endoscopically visible residual tumor. R0 resection was defined as en bloc resection with histologically free horizontal and vertical margins. Curative resection was defined as en bloc resection of tumor $\leq 10 \mathrm{~mm}$ in diameter confined to the submucosal layer, and without lymphovascular invasion. According to the time of onset, bleeding was subdivided into intraoperative and delayed bleeding [23]. Delayed bleeding was defined as hematemesis or melena that required an endoscopic hemostatic procedure using hemostatic forceps or clips [24]. Intraoperative perforation was defined as perforation occurring during the procedure. Delayed perforation was diagnosed when sudden high fever with peritoneal or retroperitoneal free air on CT occurred postoperatively, in the absence of intraoperative perforation and signs of free air on CT immediately after tumor removal [25].

\section{Follow up}

All patients received a standard intravenous dose of proton pump inhibitors (PPIs) for 3 days and were switched to oral PPIs for 4 weeks after the ESD procedure. All patients were followedup by an annual EGD to check for local recurrence and an annual CT to identify lymph node and distant metastases.

\section{Statistical analysis}

Statistical analysis was performed using JMP Pro 12 software (SAS Institute, Cary, NC). Continuous variables are presented as median and range, and categorical variables are presented as percentage. The Kaplan-Meier method was used for the analysis of long-term outcomes.

\section{Ethical approval}

This study was approved by the Institutional Review Board of Yokohama City University Medical Center (D1602024). All patients were informed of the risks and benefits of treatment before they underwent the procedure. Informed consent or alternative versions were obtained from all patients included in the study.

\section{Result}

\section{Clinicopathological characteristics}

The clinicopathological characteristics of the study cohort are summarized in Table 1. Five (63\%) of the 8 patients were men. The median age at the time of diagnosis was 69 (range 48-78) years. All patients were asymptomatic, and the tumors were detected incidentally during a screening EGD. All tumors were located in the duodenal bulb and showed 0-Is endoscopic morphology. The median tumor diameter on EUS was 6.4 (range 3-9.3) $\mathrm{mm}$. During the study period, all NAD-NETs were included in the indication of endoscopic resection (ESD or EMR) and none was treated by surgery.

\section{Short-term outcome and pathological findings}

The short-term outcome and pathological findings of all cases is summarized in Table 2. The median ESD procedural time was 43 (range 25-98) min. En bloc, R0 and curative resection were achieved in $100 \%(8 / 8), 88 \%(7 / 8)$, and $88 \%$ $(7 / 8)$ of tumors, respectively. In the single case in which an R0 resection was not achieved, the horizontal margin was negative but the vertical margin of the specimen was positive. All tumors were confined to the submucosal layer and were positive for chromogranin A and/or synaptophysin on immunohistochemical staining. The Ki-67 index was less than $2 \%$ in all tumors; therefore, all tumors were diagnosed as NET G1 based on the WHO classification. None of the tumors exhibited lymphovascular invasion. Additional surgical resection was offered to the patient in whom a curative resection was not achieved because of positive vertical margins. However, she rejected the additional treatment and underwent careful observation using EGD and CT after the ESD.

Table 1 Characteristics of patients and tumors in the present study

\begin{tabular}{lc}
\hline Characteristics & Value \\
\hline Age in years, median (range) & $69(48-78)$ \\
Sex, $\mathrm{n}(\%)$ & $5(63)$ \\
$\quad$ Male & $3(37)$ \\
$\quad$ Female & \\
Tumor location, $\mathrm{n}(\%)$ & $8(100)$ \\
$\quad$ Bulb & $0(0)$ \\
$\quad$ Descending part & $8(100)$ \\
Tumor morphology, n (\%) & $6.4(3-9.3)$ \\
\hline 0-Is & \\
Tumor size on EUS, median, mm (range)
\end{tabular}




\section{Long-term outcome}

The details of all patients in the present study are shown in Table 3. Patients were followed up for a median of 34.0 (range 18.5-62.5) months after ESD. None of the patients showed evidence of local recurrence, metachronous lymph metastasis, or distant metastasis during the follow-up period. Based on the Kaplan-Meier survival analysis, both the estimated overall survival and progression-free survival rates were $100 \%$ (Fig. not shown).

Table 2 Short and long-term outcome of patients with non-ampullary duodenal NETs who underwent ESD

\begin{tabular}{lc}
\hline Outcome & Value \\
\hline Procedural time, median, min (range) & $43(25-98)$ \\
\hline Pathological type, $\mathrm{n}(\%)$ & $8(100)$ \\
$\quad$ NET G1 & $6.5(4-9.5)$ \\
$\quad$ Tumor size on pathology, median, mm (range) & \\
\hline Invasive depth, $\mathrm{n}(\%)$ & $8(100)$ \\
$\quad$ submucosal layer & $8(100)$ \\
En bloc resection, $\mathrm{n}(\%)$ & $7(88)$ \\
R0 resection, $\mathrm{n}(\%)$ & $7(88)$ \\
Curative resection, $\mathrm{n}(\%)$ & \\
Complication, $\mathrm{n}(\%)$ & $0(0)$ \\
$\quad$ delayed bleeding & $1(13)$ \\
$\quad$ intraoperative perforation & $1(13)$ \\
delayed perforation & $34.0(18.5-62.5)$ \\
Follow-up time, median, months, (range) & $0(0)$ \\
Local recurrence, $\mathrm{n}(\%)$ & $0(0)$ \\
Lymph metastasis, $\mathrm{n}(\%)$ & $0(0)$ \\
Distant metastasis, $\mathrm{n}(\%)$ & \\
\hline
\end{tabular}

ESD, endoscopic submucosal dissection; NET, neuroendocrine tumor

\section{Complications}

Intraoperative perforation occurred in 1 patient (13\%). Stitching using an endoscopic clip was performed immediately after removing the tumor and emergent surgery was avoided. Delayed perforation occurred in 1 patient $(13 \%)$ on the day following ESD. In this case, free air around the duodenum was found on emergency CT. Since there was no obvious perforation site and the artificial ulcer was fully covered by the PGA sheet during emergency EGD, she was treated conservatively and avoided emergent surgery. Notably, this patient showed positive vertical margins. None of the patients developed belayed bleeding.

\section{Discussion}

In the present study, we assessed the short- and long-term treatment outcomes of ESD for NAD-NET in 8 consecutive patients; to the best of our knowledge, this is the largest number reported to date. Soga et al reported that metastasis was found in $9.8 \%$ of patients with duodenal NET larger than $10 \mathrm{~mm}$ in diameter [26]. Therefore, several guidelines recommend endoscopic resection, rather than significantly more invasive open surgery, for duodenal NETs $\leq 10 \mathrm{~mm}$ in diameter and confined to the submucosal layer, with no evidence of lymph node or distant metastasis on CT $[4,5,27,28]$. Various endoscopic resection methods, such as EMR, EMR-L, EMR-P, EMR-C, and ESD, have been reported in the treatment of duodenal NETs [7,11-14,16-18,29,30]. However, there is no consensus regarding the preferred method of endoscopic resection. We have employed ESD for NAD-NET since 2015 for the following reasons: i) intraoperative perforation requiring emergent surgery occurred in one patient who underwent EMR-L for NAD-NET in 2015; and ii) as we had previously reported, ESD was significantly superior to EMR-L for rectal

Table 3 Clinicopathological characteristics of patients with NAD-NET underwent ESD

\begin{tabular}{|c|c|c|c|c|c|c|c|c|c|c|c|}
\hline No. & $\begin{array}{l}\text { Age, } \\
\text { year }\end{array}$ & Sex & Location & $\begin{array}{l}\text { Size, } \\
\mathrm{mm}\end{array}$ & Depth & $\begin{array}{l}\text { Pathological } \\
\text { Morphology }\end{array}$ & $\begin{array}{l}\text { Procedural } \\
\text { time, min }\end{array}$ & $\begin{array}{l}\text { En bloc } \\
\text { resection }\end{array}$ & $\begin{array}{l}\mathrm{R} 0 \\
\text { resection }\end{array}$ & $\begin{array}{l}\text { Curative } \\
\text { resection }\end{array}$ & Complication \\
\hline 1 & 70 & M & Bulb, AW & 5 & SM & NET G1 & 76 & Yes & Yes & Yes & No \\
\hline 2 & 55 & $\mathrm{~F}$ & Bulb, AW & 5 & SM & NET G1 & 98 & Yes & Yes & Yes & No \\
\hline 3 & 48 & M & Bulb, AW & 6 & SM & NET G1 & 35 & Yes & Yes & Yes & No \\
\hline 4 & 77 & M & Bulb, SW & 7 & SM & NET G1 & 30 & Yes & Yes & Yes & No \\
\hline 5 & 67 & $\mathrm{~F}$ & Bulb, AW & 4 & SM & NET G1 & 57 & Yes & $\begin{array}{l}\text { No } \\
\text { (pVM1) }\end{array}$ & $\begin{array}{l}\text { No } \\
\text { (pVM1) }\end{array}$ & Delayed perforation \\
\hline 6 & 76 & M & Bulb, AW & 8 & SM & NET G1 & 31 & Yes & Yes & Yes & No \\
\hline 7 & 78 & M & Bulb, AW & 7 & SM & NET G1 & 25 & Yes & Yes & Yes & $\begin{array}{l}\text { Intraoperative } \\
\text { perforation }\end{array}$ \\
\hline 8 & 66 & $\mathrm{~F}$ & Bulb, AW & 9.5 & SM & NET G1 & 50 & Yes & Yes & Yes & No \\
\hline
\end{tabular}

AW, anterior wall; ESD, endoscopic submucosal dissection; NAD-NET, non-ampullary duodenal neuroendocrine tumor; NET, neuroendocrine tumor; SM, submucosal; $S W$, superior wall; VM, vertical margin 
NETs in terms of the en bloc, R0 and curative resection rates [19].

In the present study, we observed en bloc, R0 and curative resection rates of $100 \%, 88 \%$, and $88 \%$, respectively, after ESD for NAD-NET; these were similarly favorable compared to the previously reported results for rectal NETs $(100 \%, 100 \%$, and $83 \%$, respectively). Several previous studies have shown that ESD achieves better en bloc resection, R0 resection and curative resection rates than EMR [19-21]. We had reported that in rectal NETs, the en bloc, R0 and curative resection rates were $73 \%, 63 \%$ and $50 \%$, respectively, in an EMR-L group $(\mathrm{n}=22)$, and $100 \%, 100 \%$ and $83 \%$ in an ESD group $(n=24)$ [19].

In NAD-NETs, several previous studies have reported that the en bloc, R0 and curative resection rates of ESD were higher than those of EMR, EMR-L and EMR-C [13-18]. Kim et al reported 38 patients with 41 duodenal NETs treated by EMR $(n=18)$, EMR-L $(n=16)$, EMR-P $(n=3)$, or ESD $(n=4)$. In their study, the en bloc resection rates in the EMR, EMR-L, EMR-P and ESD groups were $89 \%, 100 \%, 100 \%$ and $100 \%$, respectively, while curative resection was achieved in 56\%, 25\%, 33\% and $100 \%$ [13]. To avoid an additional, invasive surgical resection, accurate pathological evaluation of horizontal and vertical margins is important. EMR is associated with positive vertical margins, or a crush and burn effect on the resected specimen, which leads to difficulties in performing a precise pathological evaluation. Recently, endoscopic full-thickness resection (EFTR) with the full-thickness resection devise (FTRD; Ovesco Endoscopy, Tübingen, Germany) for duodenal tumors had been reported [31,32]. Bauder et al reported that complete resection rates were $80 \%$ in five subepithelial tumors treated by FTRD. Their results suggested that EFTR is effective for NAD-NET, but the evidence is insufficient because of the small sample size [32]. Therefore, at the moment, ESD is the preferred method for NAD-NET to ensure accurate pathological diagnosis and avoid additional surgical resection for residual tumor.

We also assessed the long-term outcomes of patients with NAD-NET who underwent ESD. In the present study, none of the patients showed any evidence of local recurrence, or lymph node or distant metastasis, during a median follow-up period of 34.0 (range 18.5-62.5) months. In previous studies, local recurrence occurred in $0-18 \%$ of patients with NAD-NET who underwent EMR $[7,13,14,29,30,33,34]$, whereas no local recurrences were observed in patients after ESD [13-15,18]. These studies indicated that en bloc resection and R0 resection is important for avoiding local recurrence. Similarly to our own, the results from previous studies suggest that ESD is more effective than EMR for maintaining recurrence-free survival after NAD-NET treatment.

We next assessed the complications occurring during ESD for NAD-NET. ESD for duodenal tumors was associated with a higher risk of complications, including bleeding and perforation, than EMR $[13,35,36]$. In the present study, no delayed bleeding occurred; however, intraoperative perforation and delayed perforation each occurred in 1 patient (13\%). ESD for duodenal tumors is more technically difficult than for stomach or colon lesions, because the duodenal wall is very thin and the narrow lumen leads to poor endoscopic maneuverability $[13,35,36]$. This technical difficulty is considered to be associated with the high frequency of intraoperative perforations. Two small case series of ESD for NAD-NET reported a high frequency of intraoperative perforation: 67\% (2/3) [18] and 29\% (2/5) [17]. Therefore, ESD for duodenal NETs should be performed by experienced endoscopists. Although ESD is useful for en bloc resection and R0 resection, duodenal ESD is technically difficult and is only performed in a few countries. Therefore, duodenal ESD might be unacceptable in western countries. On the other hand, the efficacy of EFTR using an FTRD for duodenal superficial tumors has been reported [31,32]. Since the evidence of EFTR for duodenal NETs is still unclear, comparative studies of the efficacy and safety of ESD and EFTR are needed. In addition, exposure of the artificial ulcer after ESD to bile and pancreatic juice can also lead to delayed perforation [35]. Closure with an endoscopic clip is commonly used for the mucosal defect after ESD. However, in ESD for duodenal bulbus, closure with the endoscopic clip is technically difficult because of the narrow working space and proximity to the pylorus. Therefore, we selected a PGA sheet with fibrin glue for covering the artificial ulcer after ESD in most cases of the present study. Coverage by a PGA sheet with fibrin glue has been shown to be helpful for prevention of delayed perforation after duodenal ESD [37,38]. However, delayed perforation did occur in 1 patient who received PGA sheet coverage with fibrin glue in the present study. Therefore, careful observation of the clinical course after ESD and rapid assessment with blood tests and CT scan are needed if delayed perforation is suspected.

The patients in the present study were followed-up by CT scan for detecting metastasis. The European Neuroendocrine Tumors Society (ENETS) consensus guideline suggests that somatostatin receptor scintigraphy and 68Ga-DOTA-NOC positron emission tomography/CT are useful for diagnosis; however, this modality can lead to follow-up effectiveness. The evidence for these modalities is still insufficient and a study of a larger cohort over a longer term is needed.

The present study has several limitations. First, it was a single-center, retrospective study that assessed the outcome of ESD for NAD-NET. Second, the present study had a small number of patients. However, to the best of our knowledge, this is the largest number of patients undergoing ESD for NAD-NET reported to date. Third, the follow-up period in the present study is somewhat insufficient for detecting metastasis. Considering the rarity of NAD-NET in the general population, a multicenter study involving a larger number of patients with a long follow-up period is needed.

In conclusion, the present study showed that if there is neither lymph node nor distant metastasis evident on CT, ESD is effective and safe for NAD-NETs measuring $\leq 10 \mathrm{~mm}$ in size and confined to the submucosal layer. En bloc resection was achieved in all cases and $\mathrm{R} 0$ resection in most cases, and there were no recurrences during the follow-up period. However, further multicenter, prospective studies involving larger numbers of patients are needed to assess the efficacy and safety of ESD and to determine the preferred endoscopic resection method in patients with NAD-NETs. 


\section{Summary Box}

\section{What is already known:}

- Endoscopic resection is recommended for nonampullary duodenal neuroendocrine tumors (NAD-NETs) $\leq 10 \mathrm{~mm}$ in diameter without lymphovascular invasion

- Previous studies have reported that endoscopic submucosal dissection (ESD) was superior to endoscopic mucosal resection for achieving en $b l o c, \mathrm{R} 0$ and curative resection of rectal NETs

- However, the efficacy and safety of ESD for NADNETs remains unclear

\section{What the new findings are:}

- In NAD-NETs treated by ESD, the rates of en bloc and curative resection, and histologically free margins were sufficiently high

- Intraoperative and delayed perforation occurred in a few cases, but no emergency surgery was needed

- ESD is an efficacious and safe treatment for NADNET

\section{References}

1. Modlin IM, Sandor A. An analysis of 8305 cases of carcinoid tumors. Cancer 1997;79:813-829.

2. Modlin IM, Lye KD, Kidd M. A 5-decade analysis of 13,715 carcinoid tumors. Cancer 2003;97:934-959.

3. Yao JC, Hassan M, Phan A, et al. One hundred years after "carcinoid": epidemiology of and prognostic factors for neuroendocrine tumors in 35,825 cases in the United States. J Clin Oncol 2008;26:3063-3072.

4. Delle Fave G, O'Toole D, Sundin A, et al; Vienna Consensus Conference participants. ENETS Consensus Guidelines Update for Gastroduodenal Neuroendocrine Neoplasms. Neuroendocrinology 2016;103:119-124.

5. Lipiński M, Rydzewska G, Foltyn W, et al. Gastroduodenal neuroendocrine neoplasms, including gastrinoma - management guidelines (recommended by the Polish Network of Neuroendocrine Tumours). Endokrynol Pol 2017;68:138-153.

6. Dasari BVM, Al-Shakhshir S, Pawlik TM, et al. Outcomes of surgical and endoscopic resection of duodenal neuroendocrine tumours (NETs): a Systematic review of the literature. J Gastrointest Surg 2018;22:1652-1658.

7. Gincul R, Ponchon T, Napoleon B, et al. Endoscopic treatment of sporadic small duodenal and ampullary neuroendocrine tumors. Endoscopy 2016;48:979-986.

8. Kumta NA, Desai A, Doshi R, Kahaleh M, Sharaiha RZ. Endoscopic mucosal resection of duodenal carcinoid. Endoscopy 2016;48(Suppl 1):E158-E159.

9. Harada H, Suehiro S, Shimizu T, Katsuyama Y, Hayasaka K, Ito $\mathrm{H}$. Ligation-assisted endoscopic submucosal resection with circumferential mucosal incision for duodenal carcinoid tumor. World J Gastroenterol 2015;21:10041-10044.

10. Neumann H, Ramesh J, Wilcox CM, Mönkemüller K. Resection of carcinoids in the duodenal bulb using the band ligation technique with the Duette mucosectomy device. Endoscopy 2013;45(Suppl 2 UCTN):E365-E366.

11. Otaki Y, Homma K, Nawata Y, Imaizumi K, Arai S. Endoscopic mucosal resection with circumferential mucosal incision of duodenal carcinoid tumors. World J Gastrointest Endosc 2013;5:197-200.

12. Karagiannis S, Eshagzaiy K, Duecker C, Feyerabend B, Mozdzanowski E, Faiss S. Endoscopic resection with the cap technique of a carcinoid tumor in the duodenal bulb. Endoscopy 2009;41(Suppl 2):E288-E289.

13. Kim GH, Kim JI, Jeon SW, et al; Korean College of Helicobacter and Upper Gastrointestinal Research. Endoscopic resection for duodenal carcinoid tumors: a multicenter, retrospective study. J Gastroenterol Hepatol 2014;29:318-324.

14. Kim SH, Park CH, Ki HS, et al. Endoscopic treatment of duodenal neuroendocrine tumors. Clin Endosc 2013;46:656-661.

15. Li QL, Zhang YQ, Chen WF, et al. Endoscopic submucosal dissection for foregut neuroendocrine tumors: an initial study. World J Gastroenterol 2012;18:5799-5806.

16. Matsumoto S, Miyatani H, Yoshida Y. Endoscopic submucosal dissection for duodenal tumors: a single-center experience. Endoscopy 2013;45:136-137.

17. Matsumoto S, Miyatani H, Yoshida Y, Nokubi M. Duodenal carcinoid tumors: 5 cases treated by endoscopic submucosal dissection. Gastrointest Endosc 2011;74:1152-1156.

18. Suzuki S, Ishii N, Uemura M, et al. Endoscopic submucosal dissection (ESD) for gastrointestinal carcinoid tumors. Surg Endosc 2012;26:759-763.

19. Kaneko H, Hirasawa K, Koh R, et al. Treatment outcomes of endoscopic resection for rectal carcinoid tumors: an analysis of the resectability and long-term results from 46 consecutive cases. Scand J Gastroenterol 2016;51:1489-1494.

20. Lee DS, Jeon SW, Park SY, et al. The feasibility of endoscopic submucosal dissection for rectal carcinoid tumors: comparison with endoscopic mucosal resection. Endoscopy 2010;42:647-651.

21. Park HW, Byeon JS, Park YS, et al. Endoscopic submucosal dissection for treatment of rectal carcinoid tumors. Gastrointest Endosc 2010;72:143-149.

22. Bosman F CF. World Health Organization Classification of Tumours, Pathology and Genetics of Tumours of the Digestive System. Lyon: IARC Press 2010.

23. Oda I, Suzuki H, Nonaka S, Yoshinaga S. Complications of gastric endoscopic submucosal dissection. Dig Endosc 2013;25(Suppl 1):71-78.

24. Hoteya S, Kaise M, Iizuka T, et al. Delayed bleeding after endoscopic submucosal dissection for non-ampullary superficial duodenal neoplasias might be prevented by prophylactic endoscopic closure: analysis of risk factors. Dig Endosc 2015;27:323-330.

25. Inoue $\mathrm{T}$, Uedo $\mathrm{N}$, Yamashina $\mathrm{T}$, et al. Delayed perforation: a hazardous complication of endoscopic resection for non-ampullary duodenal neoplasm. Dig Endosc 2014;26:220-227.

26. Soga J. Early-stage carcinoids of the gastrointestinal tract: an analysis of 1914 reported cases. Cancer 2005;103:1587-1595.

27. Dalenbäck J, Havel G. Local endoscopic removal of duodenal carcinoid tumors. Endoscopy 2004;36:651-655.

28. Delle Fave G, Kwekkeboom DJ, Van Cutsem E, et al; Barcelona Consensus Conference participants. ENETS Consensus Guidelines for the management of patients with gastroduodenal neoplasms. Neuroendocrinology 2012;95:74-87.

29. Oono Y, Shinmura K, Hori K, et al. Endoscopic submucosal resection using a ligation device without injection for duodenal neuroendocrine tumors. Surg Endosc 2019;33:2008-2014.

30. Mahmud N, Tomizawa Y, Stashek K, Katona BW, Ginsberg GG, Metz DC. Endoscopic resection of duodenal carcinoid tumors: a single-center comparison between simple polypectomy and 
endoscopic mucosal resection. Pancreas 2019;48:60-65.

31. Schmidt A, Meier B, Cahyadi O, Caca K. Duodenal endoscopic full-thickness resection (with video). Gastrointest Endosc 2015; 82:728-733.

32. Bauder M, Schmidt A, Caca K. Endoscopic full-thickness resection of duodenal lesions-a retrospective analysis of 20 FTRD cases. United European Gastroenterol J 2018;6:1015-1021.

33. Min BH, Kim ER, Lee JH, et al. Management strategy for small duodenal carcinoid tumors: does conservative management with close follow-up represent an alternative to endoscopic treatment? Digestion 2013;87:247-253.

34. Park SB, Kang DH, Choi CW, Kim HW, Kim SJ. Clinical outcomes of ligation-assisted endoscopic resection for duodenal neuroendocrine tumors. Medicine (Baltimore) 2018;97:e0533.
35. Honda T, Yamamoto H, Osawa H, et al. Endoscopic submucosal dissection for superficial duodenal neoplasms. Dig Endosc 2009;21:270-274.

36. Hoteya S, Furuhata T, Takahito T, et al. Endoscopic submucosal dissection and endoscopic mucosal resection for non-ampullary superficial duodenal tumor. Digestion 2017;95:36-42.

37. Takimoto K, Imai Y, Matsuyama K. Endoscopic tissue shielding method with polyglycolic acid sheets and fibrin glue to prevent delayed perforation after duodenal endoscopic submucosal dissection. Dig Endosc 2014;26(Suppl 2):46-49.

38. Takimoto K, Toyonaga T, Matsuyama K. Endoscopic tissue shielding to prevent delayed perforation associated with endoscopic submucosal dissection for duodenal neoplasms. Endoscopy 2012;44(Suppl 2 UCTN):E414-E415. 